\title{
The Estimation of Nodal Power Supply Reliability through the Network Connectivity by Complex Network Method
}

\author{
Yuhuan Wu, Hui Ren, He Jiang, Nannan Jiang \\ North China Electric Power University, \\ Baoding, China \\ dahui.ren@,outlook.com
}

\begin{abstract}
The paper studies the reliability of the power system from the perspective of node loads. The reliability of the whole system can be estimated by evaluating the power supply reliability of each node. A measure, "connectivity" observed at load node (Ci), is proposed. $C_{i}$ is calculated through a recursion equation by evaluating the generation capacity that can be transferred from the further neighbor to the nearest neighbor of load node i. IEEE-30 bus system is taken as a test system. We calculated the index of 7 load nodes at 2 different load levels with different $N-1$ failures. The test results show that the variation of the index and that of the percentage load shedding at selected load nodes show good consistency.
\end{abstract}

\section{Introduction}

Since the happening of the large cascading blackouts in 2003 in USA and Canada, researchers worldwide and from various backgrounds (such as power system, mathematics, statistic physics, nonlinear dynamics, etc.) have performed studies with various perspectives on the evolution and intervention of cascading blackouts.

Based on the simulation or monitored and recorded data of the system, these studies focused on the whole system and meaningful progress have been obtained in understanding the mechanism of large blackouts [1, 2], risk analysis and the prevention and mitigation of the propagation of cascading failures [3]. One of the focus of the study is to predict when the system is going to have smaller load shedding, while when the system is going to suffer larger blackouts. Polymeneas and Sakis Meliopoulos [4] defined a margin-based index to find the most affecting contingency. CotillaSanchez and Hines [5] proposed a method to give early warning on voltage collapse using nodal voltage or phase angle measurement. Koç etc. [6] defined an

\author{
David Watts \\ Pontificia Universidad Católica de Chile, \\ Santiago, Chile \\ dwatts@ing.puc.cl
}

Entropy-based index to quantify the robustness of power grids against cascading failure.

Ref. [7][8] pointed out that strong connectivity and homogeneity promote higher local reliability. Along with the propagation of failure, the connectivity of the system will be gradually compromised. The capability of eliminating the local failure through the subsidiary inputs from the broader system will be damaged.

Traditional methods looked at the problem from the perspective of system to multiple lines and nodes. Because of the heterogeneity of the system due to the distribution of demand and resources, accurate estimation of the scale of power loss is difficult. If we look at the problem from the perspective of load node, the problem is simpler. If the demand at load node can be served depends on the electricity the load node can get from its nearby generator and remote generators through transmission network. Therefore, assessing nodal power supply reliability through the evaluation of the connectivity near load nodes and the remote system among load nodes and generators is a viable method. Through quick estimation of the nodal power supply reliability under each operational state, not only the scale of the outages of the system can be accurately assessed, but also the location of power outages can be identified.

The remaining of this paper is organized as follows: In section II, we provide a brief review of studies related to power system's connectivity. In section III, we give a detail introduction our method. In section IV, the IEEE 30 test system is taken as an example and the effectiveness of the method is analyzed under different operation conditions. In section IV, we summarize the contributions of this paper and explaining avenues of future work.

\section{Review on studies related to power system connectivity}

The connection of power system's topology and the capability of local failures' spreading out into a systemic one are investigated by complex networks 
metrics from global properties, such as algebraic connectivity [9], spectral radius [10] and the effective graph resistance [9][10]. Koç etc. in [10] investigate the impact of the topology of a power grid on phase transitions in its robustness by a group of metrics. Experimental results from a model of cascading failures in power grids on the IEEE power systems demonstrate the applicability of these metrics to design/optimize a power grid topology for an enhanced phase transition behavior of the system. Hines etc. [11] derived a measure of "electrical centrality" for AC power networks to describe the structure of the network as a function of its electrical topology rather than its physical topology.

In order to reflect the effect of the distribution of generators to the robustness of power system, Zhang and Tse [12] defined a measure, average effective resistance (distance) to a nearest generator of all consumer nodes (DG), to evaluate the accessibility to generators of all consumers.

$$
\begin{gathered}
\mathrm{DG}=\frac{1}{n-g} \sum_{i \in N \backslash G} d(i) \\
d(\mathrm{i})=\min \left\{R_{i s}, s \in G\right\} \\
R_{i s}=\frac{\theta_{i}-\theta_{s}}{P_{S}}
\end{gathered}
$$

where $R_{i s}$ is the effective resistance between node $i$ and node $s$.

System's topology and the configuration of resources set the inherent reliability level of power system. Normally at the transmission level, the risk of the outages of the system is evaluated through the perspective of the system as a whole. In our study, we look at the reliability of the whole system as a combination of the reliability of each node and start from the evaluation of the nodal power supply reliability of nodes.

\section{The "connectivity" among a load node and generators observed at load node}

Our work is inspired by [12]. The index given by (1) in [12] evaluates the "accessibility" through the minimal distance between a generator and a load. It is still from a system point of view and has the disadvantage of omitting the contribution of the connected network on the power supply of the node.

Looking into the network from a given load node, it has its nearest neighbors, which are defined as the Level 1 nodes. It has its second neighbors, which are defined as the Level 2 node, and so on. Lines are connecting nodes at adjacent levels.

Fig. 1 gives an illustration of the connection between a load node $D_{i}$ to other nodes and generators if looking into the network through $D_{i}$. Active power is transferred from generators at each level to nodes at nearer level through lines connecting nodes at adjacent levels until node $D_{i}$. By evaluating the "connectivity" between adjacent levels, we can finally estimate the percentage of load shedding at $D_{i}$.

\subsection{The "Connectivity" between nodes at adjacent levels of a given node load}

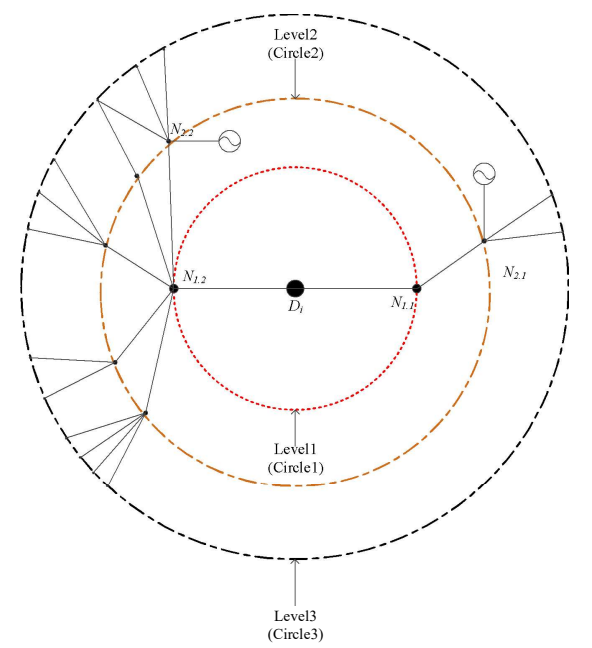

Fig.1 The connection of load node $D_{i}$ and its neighbors with different "distance"

The "Connectivity" between nodes at adjacent levels of a given node load is defined as the per unit active power that can be transferred from Level $j$ to Level $j$-1 as given in (4)-(6).

where

$$
C_{i, L=j}=C_{i, L=j}^{G} \cdot C_{i, L=j}^{T}
$$

$$
\begin{gathered}
C_{i, L=j}^{G}=C_{i, L=j+1}+\frac{\left(\sum_{L=j, k=1}^{k=m} G_{k}\right)}{\left(D_{\Sigma}-\sum_{L=j, k \neq i} D_{k}\right)} \\
C_{i, L=j>1}^{T}=\frac{\sum_{L=j, k=1}^{n} F_{k}}{\left(D_{\Sigma}-\sum_{L=j, k \neq i} D_{k}\right)} \\
C_{i, L=1}^{T}=\frac{\sum_{L=j, k=1}^{n} F_{k}}{D_{i}+D_{d s}}
\end{gathered}
$$

where $\sum_{L=j, k=1}^{k=m} G_{k}$ is the sum of the generations obtained at Level $j$. $G_{k}$ is the "effective generation" of a generator, not the installed capacity of a generator. "Effective generation" is the active power that a generator could send out through the outlets connecting at the generator node after it supply the local load. Through this definition, the deficiency of generator capacity due to the outage of the generator's outlets and its effect on the reliability of nodal power supply of the load is considered. $\sum_{L=j, k \neq i} D_{k}$ is the sum of load drown off from nodes at Level $j . F_{k}$ is the capacity limit of the $k^{\text {th }}$ line connecting nodes at Level $j$ and Level $j-1 . D_{\Sigma}$ is the total demand of the 
system. $D_{\Sigma}-\sum_{L=j, k \neq i} D_{k}$ is the remaining demand after the demand being supplied at Level $j$. Eq. (6) is the per unit transmission capacity that can be obtained by the remaining nodes at level $j(j>1)$. Lines connecting $D_{j}$ and its nearest neighbors mainly supply power to $D_{i}$. However, under some failures, the load connected to nodes at $D_{i}$ 's level 1 could only be powered through $D_{i}$. Therefore, $C_{i, L=1}^{T}$ at level 1 is given by (7), where $D_{d s}$ is the extra burden of $D_{i}$ because of the changes of the topology. Under these circumstances, $D_{d s}$ shares the transmission capacity of level 1 with $D_{i}$. Example is given in Section 4 to verify the validity of (7).

Eq.(4) is a recursion formula. The "connectivity" that observed at load node $D_{i}$ is defined as $C_{i}$ as given in (8). It can be calculated through the recursion formula (4) from further level to the nearest neighbor of $D_{i}$.

$$
C_{i}=m_{1} \cdot C_{L=1}
$$

where $m_{1}$ is the weight factor, reflecting the correlation among different load nodes, which is introduced in next subsection.

Eq.(4)-(8) only involve system's topology, line capacity limits, the capacity of generators, and the demand at load nodes. The neighbors of load node at different levels can be found out through simple network calculation. No complicated mathematics are needed, which makes the method applicable in real time operation.

\subsection{The correlation among different load nodes}

When different load nodes have common nodes of their nearest neighbors, the "connectivity" observed at Level 1 will be shared among these load nodes. Fig. 3 gives an illustration.

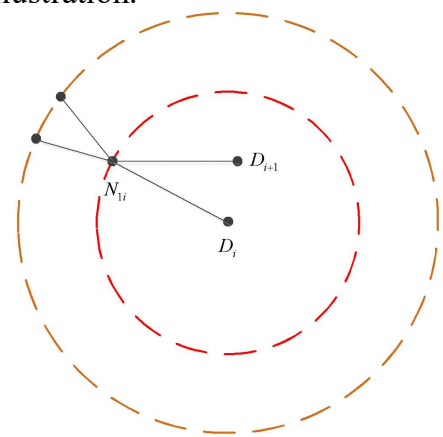

Fig. 2 the condition when $m_{1}<1$ when the nearest neighbor node $N_{1 i}$ of $D_{i}$ also supply power to other load node.

As given in Fig.3, node $N_{1 i}$ supply electricity to load node $D_{i}$ and $D_{i+1}$ at the same time. The "distance", measured as the no. of line segments, between $N_{l i}$ and
$D_{i}$ and $N_{l i}$ and $D_{i+1}$ are the same. Then the weight factor $m_{i}$ is defined as the following.

$$
m_{1}=\frac{D_{i}}{\sum_{n n, i} D}
$$

where $\sum_{n n, i} D$ is the sum demand whose distance from node $D_{i}$ is 1 line segment.

Other situations, such as load nodes connected to the nearest neighbors of $D_{i}$, are considered in (5).

For the simplicity of calculation, the effect of line reactance on the weight factor $m_{1}$ is not considered in this paper. Because we want to estimate the possible maximum "connectivity" that the load node can observe, we use transmission line limits instead of simultaneous flows and do not consider the direction of flows. We will analyze if these simplification will compromise the proposed method in the next section.

\section{Case study}

We take IEEE-30 bus system as a test system. IEEE-30 system has 4 generator nodes, 18 load nodes, and 2 hybrid nodes with both generator and load. The load at hybrid nodes has the highest power supply reliability. When no generator failure is considered, load at the hybrid nodes will not be shed, as long as the generator at the hybrid node has a larger capacity than the demand at the same node, such as load at node 2 and node 23. Therefore, we do not evaluate the power supply reliability of node 2 and node 23 .

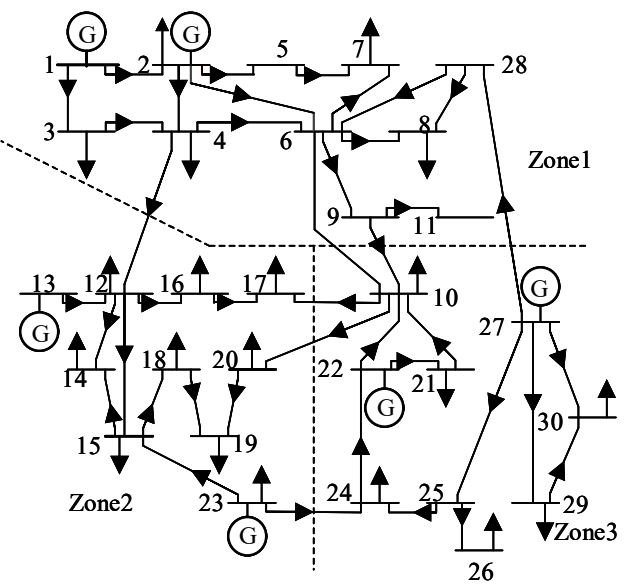

Fig. 3 IEEE-30 bus test system

In this paper, we test the effectiveness of the "connectivity" given by (8) with several cases. Under each case, the "connectivity" observed at each load node of a group of load nodes is calculated. The nodal percentage load shedding (Load-shedding at node $i$ divided by the original demand at node $i$ ) at these 
nodes are evaluated by the flow chart given in Fig.4. Fig. 5 gives the Expected Energy Not Served (EENS) of IEEE-30 system with standard parameters by the flow chart of Fig.4. According to this, we choose two load levels (load/capacity $=0.56$ and 0.8$)$ and two line failures to construct 4 cases for the further analysis. Case 1: Load level is 0.56 . No trips in the system.

Case 2: Load level is 0.8 . The line capacity limit is the same as in Case 1. No trips in the system.

Case 3: Load level is 0.8 . The line capacity limit is the same as in Case 1. Line 4-12 is tripped.

Case 4: Load level is 0.8 . The line capacity limit is the same as in Case 1. Line 2-6 is tripped.

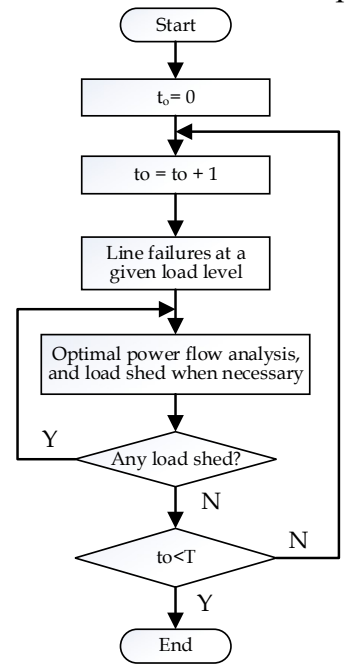

Fig. 4 Simulation flow chart for calculating the nodal percentage load shedding

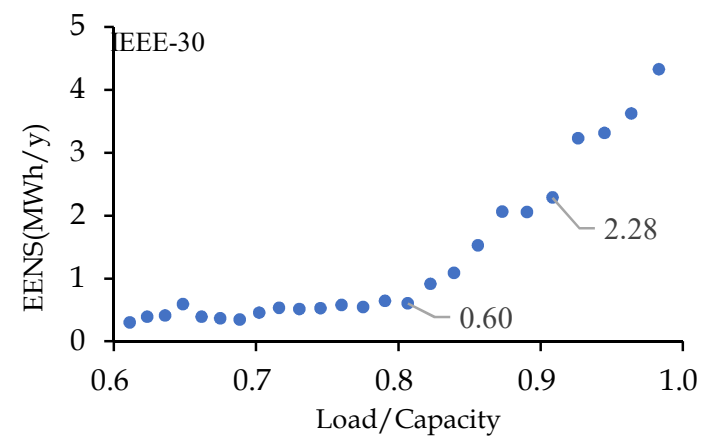

Fig.5 EENS as load/capacity increases

We trip 2 lines with largest betweenness to compromise the connectivity of the system. The betweeness of line $i$ or node $i$ is defined as the number of shortest paths between a pair of nodes through line $i$ or node $i$. Betweeness gives an information of the importance of the line or the node [13]. In power system application, the path with the smallest reactance is regarded as the shortest path. Research shows that the larger the line's betweenness, the trip of the line will affect the reliability of the system the most [13].

\subsection{Nodes with different local topology}

Since the local connection of a load node affect its connectivity more than the connection of far away, we first analyze the connectivity of two load nodes with different local connections, its variation according to different line failures near the load nodes and the correspondent nodal percentage load shedding.

Node 29 and Node 19 are taken as examples. Fig. 6(a) and Fig.7(a) give their local connections. Node 29, 27 and 30 are connected into a loop. Node 19 is served through 2 lines connected to 2 key nodes (node 15 and node 10) in zone 2 and zone 3.

The proposed method need to identify the nodes at each level of a given load node $D_{i}$. A node could be $D_{i}$ 's Level $j$ node and Level $j+1$ node at the same time when loop exists connecting $D_{i}, D_{i}$ 's Level $j$ node, and $D_{i}$ 's Level $j+1$ node. In this case, we only treat the node in the loop as $D_{i}$ 's Level $j$ node, and no node is counted more than once. Node 29 is the case and a loop exists connecting node 29, 27 and 30 as given in Fig.6(a).

When Line 27-29 fails, node 27 becomes node 29's Level 2 node, while when there is no failure, it is node 29's Level 1 node. Table I gives its Level 1, 2 and 3 nodes under different local failures. In addition, when line 27-30 fail, the demand at node 30 is served through node 29 , which means that the electricity transferred from 27 to 29 is shared by demand at node 29 and node 30 . This is the reason why we define $C_{i, L=1}^{T}$ as (7).

Table I

The topological information of Node 29 under different failures

\begin{tabular}{ccl}
\hline $\begin{array}{c}\text { Operational } \\
\text { conditions }\end{array}$ & Level & Nodes at each level \\
\hline No failures & L1 & 27,30 \\
& L2 & 25,28 \\
& L3 & $24,26,8,6$ \\
$29-30$ fails & L1 & 27 \\
& L2 & $25,28,30$ \\
& L3 & $24,26,8,6$ \\
$27-30$ fails & L1 & 27,30 \\
& L2 & 25,28 \\
& L3 & $24,26,8,6$ \\
$27-29$ fails & L1 & 30 \\
& L2 & 27 \\
& L3 & 25,28 \\
\hline
\end{tabular}




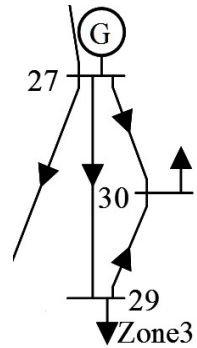

(a)

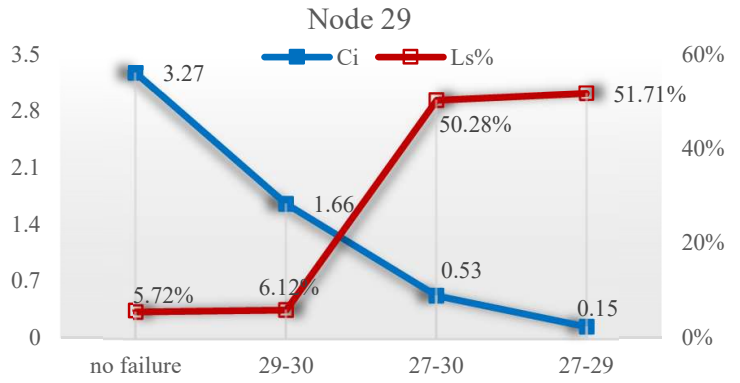

(b)

Fig. 6 (a) The local connection of Node 29; (b) Nodal percentage load-shedding v.s. $C_{i}$ under different local failures. Load level is 0.8 .

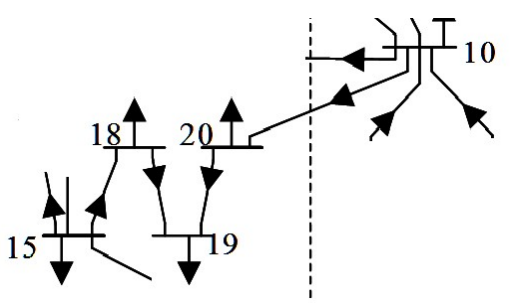

(a)

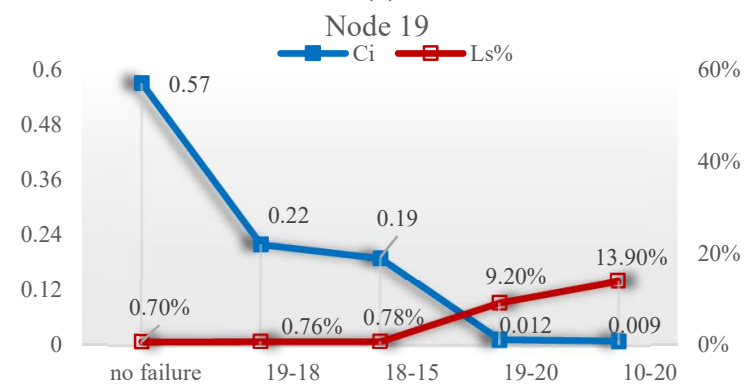

(b)

Fig. 7 (a) The local connection of Node 19; (b) Nodal percentage load-shedding v.s. $C_{i}$ under different local failures. Load level is 0.8 .

As given by Fig. 6(b) and Fig.7(b), for these two sample local connections, their connectivity $C_{i}$ and nodal percentage load shedding $L s \%$ show inverse correlation.

\subsection{The nodal power supply reliability of different nodes}

Under the same operational condition, the nodal power supply reliability of different nodes varies because of the topology of the system. We evaluate the nodal power supply reliability by the nodal percentage load shedding of load nodes. Four different load nodes, node $7,26,14$ and 15, are chosen in this subsection for further analysis under different operational conditions given in case 1 to case 4 . The results are given in Fig. 8 and Table II and III.

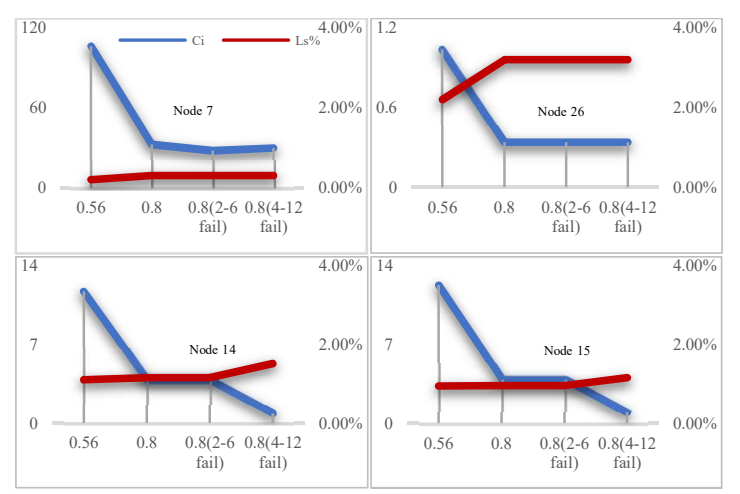

Fig.8 Comparison of nodal percentage load-shedding $L s \%$ and Connectivity $C_{i}$ observed at different nodes under two load levels and two different line failures.

The demand at node 7 is the biggest among the four nodes. According to $L s \%$ of 4 nodes under 4 different cases, the nodal power supply reliability of node 7 is the highest, about $0.2 \%-0.3 \%$. The $C_{i}$ of node 7 is also the highest. The Ls\% under the listed conditions are $0.2 \%-0.3 \%$, the least comparing to other nodes. The high reliability of node 7 contributes to its closeness to 2 generators at node 1,2 and the key node of zone 1 (node 6). Under normal condition with no line failure, node 7 has 2 level 1 nodes and 9 level 2 nodes as well as more than 10 lines connecting them. Therefore, it has a very high $C_{i}$ according to (4)-(7).

From the definition of (4)-(7), when the load level of the system increase, the $\mathrm{Ci}$ of each node decreases. According to the simulation result given by Fig.8, when we only improve the load level of the system from 0.56 to $0.8, C_{i}$ decreases as load level increases. However, the $L s \%$ at node 7,14 and 15 remains the same. $L s \%$ at node 26 increases.

Line failures affect different nodes differently. The trip of line 2-6 does not cause extra load shedding at these 4 nodes, because there are multiple extra routes between node 2 and node 6 and failure of line 2-6 does not affect the transferring of electricity from node 2 to 6.

The trip of line 2-6 and 4-12 cause the same load shedding at node 7 . Node 26 is the same case. 
However, the trip of line 4-12 affect node 14 and 15 more than the trip of line 2-6, because node 14 and 15 obtain electricity from zone 1 through line $4-12$. The changes of $C_{i}$ of these nodes under the 2 line failures correspond to the changes of $L s \%$ at these nodes.

The $C_{i}$ of node 7 is far more than that of node 14 and 15 , and the $C_{i}$ of node 26 is the least. We need to remember that the $L s \%$ refers to the nodal percentage load shedding. Even though the $L s \%$ of different nodes is the same, the load shedding at nodes could be different because of the differences of the original demands at these nodes. According to the load demand at these 4 nodes given in Table II, we can see that the demand at node 7 is the highest, while that at node 26 is the least. In general, higher reliability guaranteed by power system design should be given to important or higher demand. The design with higher reliability means easier accessibility to more generations through multiple routes - This is what we try to reflect in the design of $C_{i}$. More simulation results is given in Fig.9(a) and (b).

Table II

Demand of 4 nodes at 2 different load level

\begin{tabular}{lcccc}
\hline & 7 & 26 & 14 & 15 \\
\hline $\mathrm{L}=0.56$ & 22.8 & 3.5 & 6.2 & 8.2 \\
$\mathrm{~L}=0.8$ & 32.52 & 5 & 8.86 & 11.71 \\
\hline
\end{tabular}

Table III

$L s \%$ and $C i$ observed at different nodes and Initial load of node under two load levels and two different line failures.

\begin{tabular}{crrrr}
\hline & $\mathrm{L}=0.56$ & $\mathrm{~L}=0.8$ & $\begin{array}{r}\mathrm{L}=0.8 \\
2-6 \text { fail }\end{array}$ & $\begin{array}{r}\mathrm{L}=0.8 \\
4-12 \text { fail }\end{array}$ \\
\hline$C_{i}$ & 106.22 & 32.48 & 27.74 & 29.60 \\
$L s \%$ & $0.2 \%$ & $0.3 \%$ & $0.3 \%$ & $0.3 \%$ \\
$C_{i}$ & 1.04 & 0.34 & 0.34 & 0.34 \\
$L s \%$ & $2.2 \%$ & $3.2 \%$ & $3.2 \%$ & $3.2 \%$ \\
$C_{i}$ & 11.75 & 3.87 & 3.87 & 0.87 \\
$L s \%$ & $1.1 \%$ & $1.15 \%$ & $1.15 \%$ & $1.52 \%$ \\
$C_{i}$ & 12.3 & 3.96 & 3.96 & 0.98 \\
$L s \%$ & $0.95 \%$ & $0.96 \%$ & $0.96 \%$ & $1.16 \%$ \\
\hline
\end{tabular}

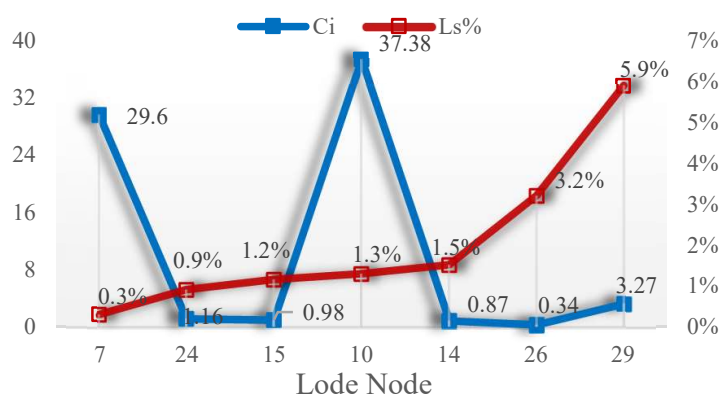

(a)

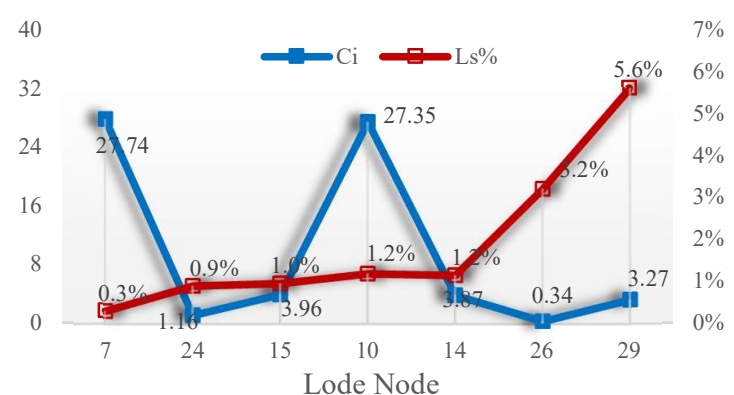

(b)

Fig.9 Comparison of nodal percentage load shedding $L s \%$ and connectivity $C_{i}$ at load level 0.8 when 4-12 failed (a), 2-6 failed (b)

This is the very first attempt to study the transmission system reliability through nodal power supply reliability than a systemic index. The features and shortcomings of the proposed method are concluded in the following.

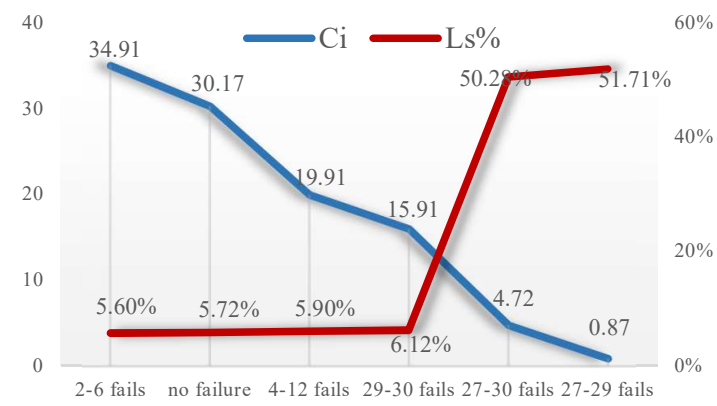

(a)

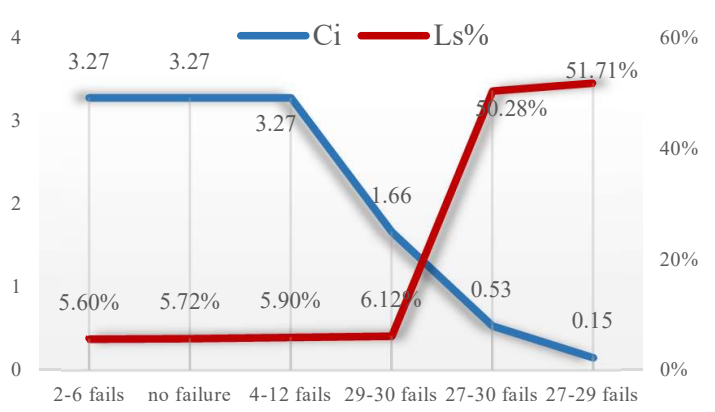

(b)

Fig. $10 C_{i}$ and $L s \%$ of node 29 under different failures at load level 0.8. (a) $C_{i}$ is calculated until level 6 to go through all generators. (b) $C_{i}$ is calculated until level 3 .

1) The $C_{i}$ of each node is decided by the "depth" of the observation. The $C_{i}$ considering up to level 3 or level 6 of a given load node will be different. When less level is included in the calculation of $C_{i}$, the influence of a failed line far away from the studied load node will not be reflected in the $C_{i}$. In this paper, except for Fig.10(a), all $C_{i}$ provided in figures and tables are calculated until level 3 . Fig 10 is an example 
to show the difference of $C_{i}$ until level 6 to go through all generators and $C_{i}$ until level 3 . We can see the inverse correlation between $C_{i}$ and nodal load shedding hold in Fig. 10 (a) and (b). $C_{i}$ is larger when deeper level is included. For smaller system, such as IEEE-30 bus system, calculation of $C_{i}$ up to level 3 or level 6 will not bring much difference at the computation efficiency. However, for larger system, a failed line far away from a load node will not bring any negative effect under some conditions, or limit the transfer of generation to loads under other conditions, such as high load level or multiple trips of critical lines. Calculating $C_{i}$ until going through all generators and lines will lower computation efficiency for larger system and is not necessary under some conditions.

2) We have not provided the coefficients between $C_{i}$ and $L s \%$ of each load node. From Fig.6-9 and Table II-III, we can see that for some nodes, there exists a threshold, below that, nodal load shedding will increase. More simulations under more conditions are needed to decide the coefficients and the threshold.

3) The $L s \%$ is decided by simulation given in Fig. 4 . The objective function and constraints of optimal power flow analysis (OPF) under each condition is given in Appendix. In the objective function of OPF, the cost function of all generators are quadratic with different parameters. The cost of load shedding at each node are also chosen to be the same. When these conditions are not hold, modifications are needed for the calculation of $C_{i}$.

4) In (4)-(7), we use the line flow limit instead of instantaneous flow to evaluate the maximum possible connectivity that can be observed by the load node. When other lines' effective transmission margin are limited because transmission bottlenecks under some failure conditions, the connectivity observed at a load node may be over-estimated. However, if the instantaneous flow is used, the connectivity could be under-estimated under other conditions. Possible solution for this is to modify (4) to (7) by using "effective" transmission capacity instead of transmission line limit in (4) to (7). Sensitivity method can be used to identify the "effective" transmission margin when transmission bottleneck exists.

\section{Conclusion}

Higher levels of connectivity between different regions improves the operational economy of the systems and often provides a more reliable power supply. On the contrary, the decrease of connectivity between load node and other part of the network could compromise the power supply reliability at the load node.
The paper studies the reliability of the power system from the perspective of the node loads. When the load shedding at each node can be estimated under any operational condition, the scale of the outages of the whole system can be estimated. A measure, "connectivity" observed at load node $\left(C_{i}\right)$, is proposed. $C_{i}$ is calculated through a recursion equation by evaluating the generation capacity that can be transferred from the further neighbor to the nearest neighbor of load node $i$. Higher $C_{i}$ at node $i$ means smaller load-shedding at load $i$ under the studied operational condition.

IEEE-30 bus system is taken as a test system. We calculated the "connectivity" observed at 7 load nodes at 2 different load levels with 2 different $\mathrm{N}-1$ failures. The test results show that variation of the connectivity and the nodal percentage load-shedding at selected load nodes show good consistency.

Because the calculation is simple, the proposed method can be used for evaluating the load-shedding of each node when there is topology changes or load variations, or for screening contingencies in real time.

Future research focus include: 1) improving the connectivity observed at a load node into local connectivity and a system connectivity. The former is evaluated by the proposed method in this paper. The latter, the connectivity of the rest of the system, could be evaluated by some methods already published. 2) Modifying the method to evaluated the connectivity properly when transmission bottleneck exists.

\section{Acknowledgment}

Hui Ren thanks Pontificia Universidad Católica de Chile for the invitation of working with Prof. David Watts. David Watts thanks CONICYT funding by FONDECYT project 1141082.

\section{References}

[1] I. Dobson, B.A. Carreras, V.E. Lynch, and D.E. Newman "An initial model for complex dynamics in electric power system blackouts", the 34th Hawaii International Conference on System Science, Hawaii, 2001.

[2] IEEE PES CAMS Task Force on Understanding, Mitigation and Restoration of Cascading Failures, "Initial review of methods for cascading failure analysis in electric power transmission systems", IEEE PES General Meeting, Pittsburgh, PA USA, 2007.

[3] IEEE Task Force on Understanding, Prediction, Mitigation and Restoration of Cascading Failures (M. Vaiman, K. Bell, Y.Chen, B. Chowdhury, I. Dobson, P. Hines, M. Papic, S. Miller, P. Zhang), "Risk assessment of 
cascading outages: methodologies and challenges", IEEE Transactions on Power Systems, vol. 27, no. 2, 2012, pp. 631-641.

[4] E. Polymeneas and A. P. Sakis Meliopoulos, "Marginbased framework for online contingency selection in unbalanced networks", IEEE Transactions on Power Systems, vol. 32, no. 1, Jan. 2017, pp. 30-38.

[5] E. Cotilla-Sanchez, P. D. H. Hines, and C. M. Danforth, "Predicting critical transitions from time series synchrophasor data", IEEE Transactions on Smart Grid, vol. 3, no. 4, Dec. 2012, pp. 1832-1840.

[6] Y. Koç, M. Warnier, R. E. Kooij, and F. M. T. Brazier, "An entropy-based metric to quantify the robustness of power grids against cascading failures", Safety Science, vol. 59, Nov. 2013, pp. 126-134.

[7] M. Scheffer, S. R. Carpenter, T. M. Lenton, J. Bascompte, W. Brock, V. Dakos, J. van de Koppel, I. A. van de Leemput, S. A. Levin, E. H. van Nes, M. Pascual, and J. Vandermeer, "Anticipating critical transitions", Science, vol. 338, no. 6105, Oct. 2012, pp. 344-348.

[8] Egbert H. van Nes, Marten Scheffer, "Implications of spatial heterogeneity for catastrophic regime shifts in ecosystems", Ecology, vol. 86, no. 7, 2005, pp. 1797-1807.

[9] Y. Koç, M. Warnier, P. Van Mieghem, R. E. Kooij, and F. M. T. Brazier, "A topological investigation of phase transitions of cascading failures in power grids", Physica A: Statistical Mechanics and its Applications, vol. 415, Dec. 2014, pp. 273-284.

[10] Y. Koç, M. Warnier, P. V. Mieghem, R. E. Kooij, and F. M. T. Brazier, "The impact of the topology on cascading failures in a power grid model", Physica A: Statistical Mechanics and its Applications, vol. 402, May 2014, pp. 169-179.

[11] P. Hines and S. Blumsack, "A centrality measure for electrical networks", in Hawaii International Conference on System Sciences, Proceedings of the 41st Annual, 2008, pp. 185-185.

[12] X. Zhang and C. K. Tse, "Assessment of robustness of power systems from a network perspective", IEEE Journal on Emerging and Selected Topics in Circuits and Systems, vol. 5, no. 3, Sep. 2015, pp. 456-464.

[13] G. A. Pagani and M. Aiello, "The Power grid as a complex network: A survey", Physica A: Statistical Mechanics and its Applications, vol. 392, no. 11, Jun. 2013, pp. 2688-2700.

\section{Appendix}

A. The model of optimal power flow in Fig.4

Optimal power flow: The objective function is the minimization of generation cost and the cost of load shedding:

$$
\min _{P_{G i}, P_{D j}} \operatorname{Cost}=\sum_{i \in G} C\left(P_{g i}\right)+\sum_{j \in D} K \times\left(\Delta P_{D j}\right)
$$

The constraints are:

$$
\begin{gathered}
\sum_{k \in i} P_{G k}-P_{D i}-U_{i} \sum_{j=1}^{n} U_{j}\left(G_{i j} \cos \theta_{i j}+B_{i j} \sin \theta_{i j}\right)=0 \\
\sum_{k \in i} Q_{G k}-Q_{D i}-U_{i} \sum_{j=1}^{n} U_{j}\left(G_{i j} \cos \theta_{i j}-B_{i j} \sin \theta_{i j}\right)=0 \\
P_{G i m i n} \leq P_{G i} \leq P_{G i m a x} \\
Q_{G i m i n} \leq Q_{G i} \leq Q_{\text {Gimax }} \\
U_{\text {imin }} \leq U_{i} \leq U_{i m a x} \\
-\overline{P_{i j}} \leq P_{i j} \leq \overline{P_{i j}} \\
-\overline{P_{j i}} \leq P_{j i} \leq \overline{P_{j i}}
\end{gathered}
$$

where $P_{G i}$ is the real generation of $i^{\text {th }}$ generator and $P_{D j}$ is the real power demand at the $i^{\text {th }}$ load node. $\Delta P_{D j}$ is the load-shedding at node $j$. The coefficient $K=100$ is a sufficiently large constant number to make sure that load shedding is performed when it is the only choice for finding a feasible solution for the optimal power flow. $C\left(P_{g i}\right)$ is the quadratic cost function of generator $i . P_{G k}$ represents the active output of the $k^{\text {th }}$ generator. $Q_{G k}$ represents the reactive output of the $k^{\text {th }}$ generator. $P_{D i}$ is the active load of the node $j . Q_{D i}$ is the reactive load of the node j. $P_{\text {Gimin }}, P_{\text {Gimax }}, Q_{\text {Gimin }}$ and $Q_{\text {Gimax }}$ are the real power output limit and reactive power output limit of generator $i$. $U_{i}, U_{j}$, and $\theta_{i j}$ are the voltage amplitude and phase difference of the node $i$ and $j . U_{\text {imin }}$ and $U_{\text {imax }}$ are voltage limits. $G_{i j}$ and $B_{i j}$ are the corresponding conductance and susceptance of line $i j$ in the network admittance matrix. $P_{i j}$ is power flow on line $i j$, and $\overline{P_{l j}}$ is the flow limit of line $i j$.

The cost function of 6 generators are given in the following table.

Table A.1

Generators' cost functions of IEEE-30 system

\begin{tabular}{cl}
\hline $\begin{array}{c}\text { Generator } \\
\text { node }\end{array}$ & \multicolumn{1}{c}{ Cost function $C\left(P_{g i}\right)$} \\
\hline 1 & $0.02 P_{g i}{ }^{2}+2 P_{g i}$ \\
2 & $0.0175 P_{g i}{ }^{2}+1.75 P_{g i}$ \\
22 & $0.0625 P_{g i}{ }^{2}+P_{g i}$ \\
27 & $0.00834 P_{g i}{ }^{2}+3.25 P_{g i}$ \\
23 & $0.025 P_{g i}{ }^{2}+3 P_{g i}$ \\
13 & $0.025 P_{g i}{ }^{2}+3 P_{g i}$ \\
\hline
\end{tabular}

